\title{
Linear Dichroism Activity of Chiral Poly(p-Aryltriazole) Foldamers
}

\author{
Jake G. Carter, Rueben Pfukwa, Liam Riley, James H. R. Tucker, Alison Rodger, Timothy R. Dafforn,* \\ and Bert Klumperman*
}

Cite This: ACS Omega 2021, 6, 33231-33237

Read Online

ACCESS | Lلw Metrics \& More | 回 Article Recommendations | sI Supporting Information

\begin{abstract}
Controllable higher-order assembly is a central aim of macromolecular chemistry. An essential challenge to developing these molecules is improving our understanding of the structures they adopt under different conditions. Here, we demonstrate how flow linear dichroism (LD) spectroscopy is used to provide insights into the solution structure of a chiral, self-assembled fibrillar foldamer. Poly(para-aryltriazole)s fold into different structures depending on the monomer geometry and variables such as solvent and ionic strength. LD spectroscopy provides a simple route to determine chromophore alignment in solution and is generally used on natural molecules or molecular assemblies such as DNA and M13 bacteriophage. In this contribution, we show that LD spectroscopy is a powerful tool in the observation of self-assembly processes of synthetic foldamers when complemented by circular dichroism, absorbance spectroscopy, and microscopy. To that end, poly(paraaryltriazole)s were aligned in a flow field under different solvent conditions. The extended aromatic structures in the foldamer give rise to a strong LD signal that changes in sign and in intensity with varying solvent conditions. A key advantage of LD is that it only detects the large assemblies, thus removing background due to monomers and small oligomers.
\end{abstract}

\section{INTRODUCTION}

Aromatic foldamers, which adopt predictable, well-defined helical conformations in solution, have significant potential for applications in biotechnology as they bear a close resemblance to natural helical systems, but are amenable to low-cost commercial scale production. ${ }^{1-5}$ They are convenient building blocks for preparing supramolecular assemblies such as nanocylinders by stacking foldamer helices into columns. 6,7 Application areas to date range from molecular recognition, $^{8-10}$ artificial ion channels, ${ }^{8,11}$ and virus mimics ${ }^{12}$ to asymmetric catalysis. ${ }^{13,14}$

A key element of the design-build-test cycle for macromolecular assemblies, such as the ones formed by foldamers, is a detailed understanding of the higher-order structures that they form. For chiral structures such as those in this study, the helical folding and self-assembly are normally characterized by $\mathrm{NMR}^{15-19}$ fluorescence, ${ }^{20,21} \mathrm{UV}$-visible absorbance, ${ }^{15}$ and circular dichroism (CD) spectroscopy ${ }^{22,23}$ for the solution state and X-ray crystallography ${ }^{16,24}$ for the solid state. The unfolded and folded oligomers/polymers give different spectroscopic signals. For example, in foldamers with aromatic moieties involved in the structure, helical folding is characterized by the upfield shifting of aromatic protons $\left({ }^{1} \mathrm{H}\right.$ NMR), ${ }^{15}$ the appearance of excimer-like fluorescence emission, ${ }^{20,21}$ or hypochromicity in absorbance spectra. If an excess in one handedness of the helical conformation is induced, a Cotton effect is observed in the CD spectrum in the chromophore region of the foldamer backbone. ${ }^{12,22,25,26}$ Further assembly of the foldamer helices into columns produces increased upfield shifts and signal broadening (via ${ }^{1} \mathrm{H}$ NMR spectroscopy) or enhanced hypochromicity (via absorbance spectroscopy), while the formation of solution stable-stacked helices can be visualized via cryo-transmission electron microscopy (cryo-TEM). ${ }^{12,23,27}$ These approaches are well-established and provide a rich description of the foldamer structure.

The poly(para-aryltriazole) $[\mathrm{P}(p$-AT $)$, Figure 1] foldamers synthesized by Pfukwa et al., ${ }^{12}$ was designed with features to promote assembly, and we found using absorbance, CD, NMR, and microscopy that they display a highly ordered selfassembly process as solvent transitioned from dimethylformamide (DMF) to water. At a low water content, the polymer adopted an unstructured random coil. As the water content was increased, a series of helical structures formed, which we described as first "loose spring", then "tight coil", and then long and thin helical columns. The assembly process was determined to be a thermodynamically controlled formation of well-defined rod-shaped nanostructures in water/DMF mixtures, with $>40 \%$ water. $^{12,28}$

Received: November 2, 2021

Accepted: November 15, 2021

Published: November 24, 2021 

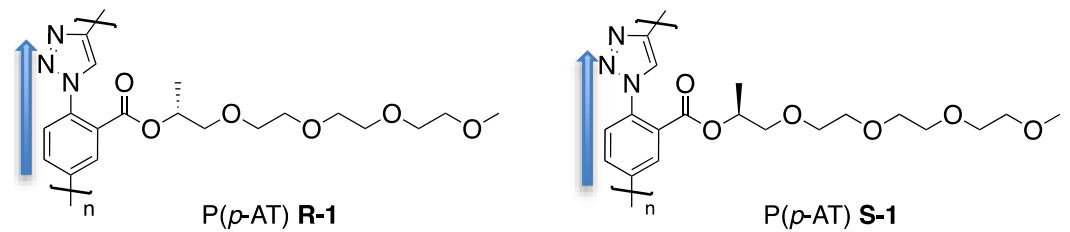

Figure 1. Structural formulae of the foldamers $\mathrm{P}(p-\mathrm{AT}) \mathrm{R}-\mathbf{1}$ and $\mathrm{P}(\mathrm{p}-\mathrm{AT}) \mathrm{S}-\mathbf{1}$. Blue arrows indicate approximate directions of EDTMs for the monomer units.

However, molecular-level details such as the relative orientations of chromophores within a structure are difficult to determine using NMR, CD, and absorbance spectroscopy. It was also unclear to what extent the measured data could be ascribed solely to the target-extended helical structures or whether monomers and oligomers were also contributing to the observed spectra. Since our previous electron microscopy (EM) images showed that the $\mathrm{P}(p$-AT $)$ foldamer assemblies possess very high aspect ratios and suggested that they might be rigid, we decided to explore what information could be deduced from flow linear dichroism (LD). LD is a measure of the difference in the absorbance of two perpendicular linearly polarized beams of light (eq 1). ${ }^{29,30}$ LD data provides a significant extra constraint on what types of molecular structures are possible, so combining microscopy, absorbance, $\mathrm{CD}$, and $\mathrm{LD}$ takes us a significant step towards being able to deduce molecular-level structures from spectroscopic and microscopic data. Specifically, CD reports on local chiral structures that cause local coupling of (electric and magnetic) transition dipole moments. Absorbance and LD are also dependent on locally coupled dipole moments for locations of bands and intensities, but LD is also dependent on the net orientation of those transition moments, so it gives information about local structures relative to the long axis of any structure.

$$
\mathrm{LD}=A_{\|}-A_{\perp}
$$

The absorbance of light is dependent on the orientation of the electron dipole transition moments (EDTMs) of the molecule relative to the electric field of the light, and therefore, the difference in absorption gives rise to a characteristic spectrum based on the identity of the chromophores and their orientation within an assembly. ${ }^{30-32}$ In most cases, the long axis of the assembly is aligned along the orientation axis. If an EDTM is oriented more parallel than perpendicular to the orientation direction, a positive LD signal is observed. On the other hand, a more perpendicular EDTM results in a negative LD signal. When the EDTM is at an angle $\alpha$ to the long axis, the LD may be expressed as shown in eq 2 .

$$
\mathrm{LD}^{r}=\frac{\mathrm{LD}}{A_{\text {iso }}}=\frac{3}{2} S\left(3 \cos ^{2} \alpha-1\right)
$$

where $L D^{r}$ is the reduced LD and $S$ is the orientation factor of the EDTM ( 1 being perfectly orientated and 0 being a random orientation). ${ }^{30,31}$ When $\alpha$ is equal to $54.7^{\circ}$, the "magic angle", the LD signal is always 0 . As preferential orientation of the EDTMs of the structure is vital for this analytical technique, the molecules making up the structure must be aligned to give a signal. The physical properties of a structure are the key to determining which method is used to orientate the molecule. Rigid or semiflexible polymers/assemblies of molecules in solution may be aligned using laminar flow with the long axis of the structure being parallel to the flow of the solution. Through the use of a micro-Couette cell made of quartz, it is possible to align microliters of the sample and permit the transmission of light to record the LD signal. ${ }^{29,32-34}$ This means flow LD spectroscopy produces strong signals for assemblies with high aspect ratios such as filamentous bacteriophage (e.g., M13) ${ }^{35}$ and DNA. ${ }^{36}$ Small molecules that are unable to be aligned through shear flow, due to an insufficient force exerted on the molecule, are instead able to be aligned through their adsorption onto a polymer film which is mechanically stretched. The stretching aligns the long axis of the molecule parallel to the stretch direction. ${ }^{29,37-39}$

While the use of LD as a tool for studying the orientation of chromophores in biopolymers such as DNA, protein assemblies, and M13 bacteriophage is well established, ${ }^{31,32,34,35,39-45}$ as far as we are aware, flow LD has extremely limited use for the analysis of synthetic polymers. This is partly because synthetic polymers do not usually form secondary and tertiary structures such as double helical DNA and coiled-coil, cross-beta, or protein fibers which enhance biomolecular polymer rigidity and hence flow orientation.

In this study, we used LD to probe the secondary and tertiary structures formed by the two chiral enantiomeric, $\mathrm{P}(p$ AT) S-1 and P(p-AT) R-1, foldamers developed by Pfukwa et al., ${ }^{12}$ which have previously been shown by CD and EM to form a range of different secondary and tertiary structures. We demonstrate that $\mathrm{LD}$ can provide unique insights into the structure and assembly process for the extended foldamer structures which can be used to optimize future foldamer designs. This data lays the foundations for the use of the foldamers in bioassays developed using LD as the detector. ${ }^{46}$

\section{RESULTS AND DISCUSSION}

We have previously seen with diphenyl alanine ${ }^{47}$ that the process of forming self-assembled structures may vary from experiment to experiment depending as it does on nucleation events. We therefore performed extensive experiments as a function of time and concentration of the monomer. Supposedly identical experiments for S-1 (or R-1) did indeed show differences in the absorbance and CD spectra observed, even though the same trends always occurred across the experiment. A typical data set is shown in Figures $2 \mathrm{~A}$ and $\mathrm{S} 1$ for $\mathrm{P}(p-\mathrm{AT}) \mathbf{S - 1}$ for experiments undertaken at similar concentrations to our previous work (Pfukwa et al. ${ }^{12}$ ). Figure $2 \mathrm{~B}$ shows the CD spectra of $\mathrm{P}(p-\mathrm{AT}) \mathrm{S}-\mathbf{1}$ and $\mathrm{P}(p-\mathrm{AT}) \mathbf{R}-\mathbf{1}$ $35 \%$ water to be mirror images, indicative of the opposite handedness of the helices in both systems. Figure S2 contains a data set for R-1 which has opposite signed CD but otherwise is similar to Figure 2A illustrating the degree of experiment to experiment variability. We previously ascribed the phases of folding apparent in Figure $2 \mathrm{~A}$ to be a random coil in DMF (red), predominantly loose helix from 10 to $20 \%$ water (orange), and a tighter helix above $20 \%$, which ultimately assembles into helical columns (fibers) above $40 \%$ (green to 

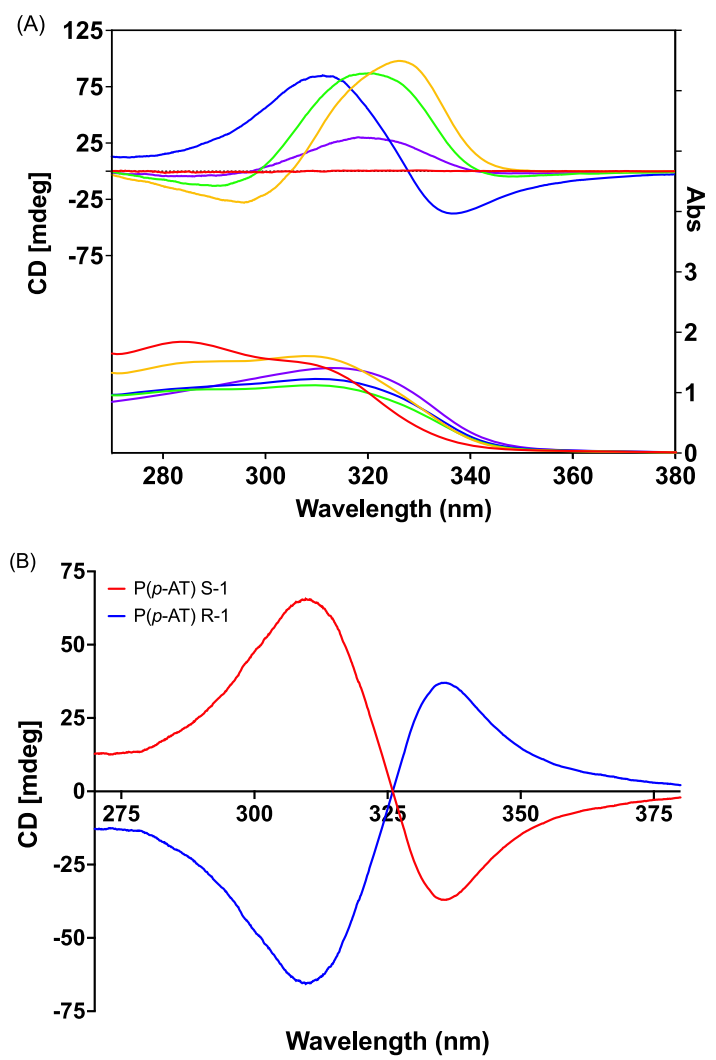

Figure 2. (A) CD (top, $0.5 \mathrm{~mm}$ path length) and absorbance (bottom, $1 \mathrm{~cm}$ path length) spectra of the solvent titrations of $\mathrm{P}(p$ AT) S-1 (10 $\mu \mathrm{M}$ monomer). (Red) $0 \%$ water, (orange) $15 \%$ water, (green) 21\% water, (blue) 29\% water, and (purple) $90 \%$ water. (B) CD spectra of $\mathrm{P}(p-\mathrm{AT}) \mathrm{S}-\mathbf{1}$ (red) and $\mathrm{P}(p-\mathrm{AT}) \mathrm{R}-\mathbf{1}$ (blue) at $10 \mu \mathrm{M}$ concentration and $35 \%$ water.

blue to purple). The goal of this work was to understand more about the assemblies formed at different water/DMF ratios.

LD of the Polymer Chromophores. As LD is based on the direction of EDTMs relative to any orientation axis, we first measured the film LD spectra of the polymer chromophores. The lowest energy transition of 1-phenyl-1,2,3-triazole (3) and 4-(1-phenyl-1H-1,2,3-triazol-4-yl)aniline (7) both showed positive signals with maxima, respectively, at 250 and 285 $\mathrm{nm}$ (Figures S3 and S4). This means that the transitions are polarized along the long axis of these molecules and thus also polarized along the long axis of the polymer backbone.

LD of Polymers as a Function of Water. Typical flow LD spectra collected in a microvolume Couette flow cell ${ }^{47,48}$ are shown in Figures 3 and S5-S7. The signs and shapes of the LD spectra are independent of the foldamer concentration between 1 and $10 \mu \mathrm{M}$ but change as a function of the water content. From 0 to $9 \% \mathrm{H}_{2} \mathrm{O}$, as expected, there is no $\mathrm{LD}$ signal since the molecularly dissolved polymers are not aligned in the laminar flow field.

$\mathrm{P}(p-\mathrm{AT})$ Assemblies Formed at $10-20 \%$ Water. The flow LD spectra for the $10-20 \%$ water $\mathrm{P}(p-\mathrm{AT})$ samples (red and orange spectra shown in Figure 3) are simpler than the corresponding $\mathrm{CD}$ and absorbance spectra of Figure 2: they show a single negative band with a maximum at $\sim 310 \mathrm{~nm}$, which corresponds to the longer wavelength component of the absorbance and CD spectra. Thus, the $10-20 \%$ water regime has two chiral populations: one that is long enough and stiff
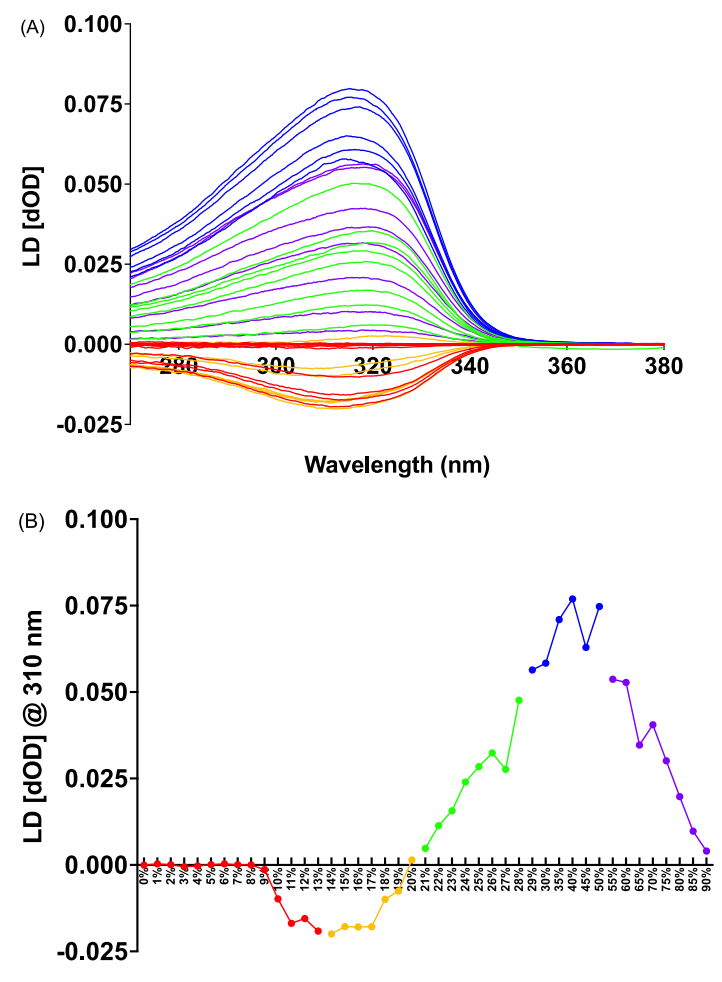

Water Percentage

Figure 3. $\mathrm{LD}$ of the solvent titrations of $\mathrm{P}(p-\mathrm{AT}) \mathrm{S}-1 \quad(10 \mu \mathrm{M}$ monomer and path length $0.5 \mathrm{~mm}$ ). (Red) $0-13 \%$ water, (orange) 14-20\% water, (green) $21-28 \%$ water, (blue) $29-50 \%$ water, and (purple) 55-90\% water. (A) Wavelength scans. (B) $310 \mathrm{~nm}$ wavelength data.

enough to align ( $310 \mathrm{~nm}$ maximum) and one that is not (295 nm maximum). ${ }^{12,48}$

A negative LD signal indicates that the corresponding EDTM is oriented more perpendicular than parallel to the long axis of the structure. Therefore, the structure adopted is one where the long axis of the monomers is $>54.7^{\circ}$ from the structure's long axis. Concomitantly, the chirality of the polymers biases their folding toward a specific handedness. The CD spectroscopy is dominated by the interactions of the backbone chromophores in that geometry to create a net electric dipole moment and a net magnetic dipole moment. Since we observe a positive CD band at $320 \mathrm{~nm}$ for the $\mathbf{S - 1}$ enantiomer, the structure must have parallel magnetic and electric dipole transition moments, thus a right-handed helix (as discussed by Nordén et al. ${ }^{31}$ ). In ref 12 , we proposed the polymer wound into a helix $\sim 3 \mathrm{~nm}$ in diameter (the diameter being determined by the curve of the monomer units) with a pitch of $\sim 10-15^{\circ}$. The cartoon of Figure $4 \mathrm{D}$ is drawn consistent with this proposal and the $\mathrm{CD} / \mathrm{LD}$ data.

Negative stain TEM (Figure 4A) images of the assemblies deposited at $15 \%$ water show curved fibers approximately 500 $\mathrm{nm}$ in length that associate into bundles (even though the bundling may be an artifact of the TEM sample preparation process). The reduced LD (eq 2 ) for the $20 \%$ water is $\sim 0.22$. If the helix pitch is $10-15^{\circ}$, as illustrated in Figure $4 \mathrm{D}$, the polymer orientation parameter $S$ is $\sim 0.09 \pm 0.02$. A $300 \mathrm{~nm}$ rigid fiber of $\sim 3 \mathrm{~nm}$ width is expected to have $S \sim 0.2,{ }^{49}$ whereas the quite flexible DNA (of width $2.5 \mathrm{~nm}$ ) has $S \sim 0.01$ for a $500 \mathrm{~nm}$ polymer. Therefore, the LD data for $10-20 \%$ are 

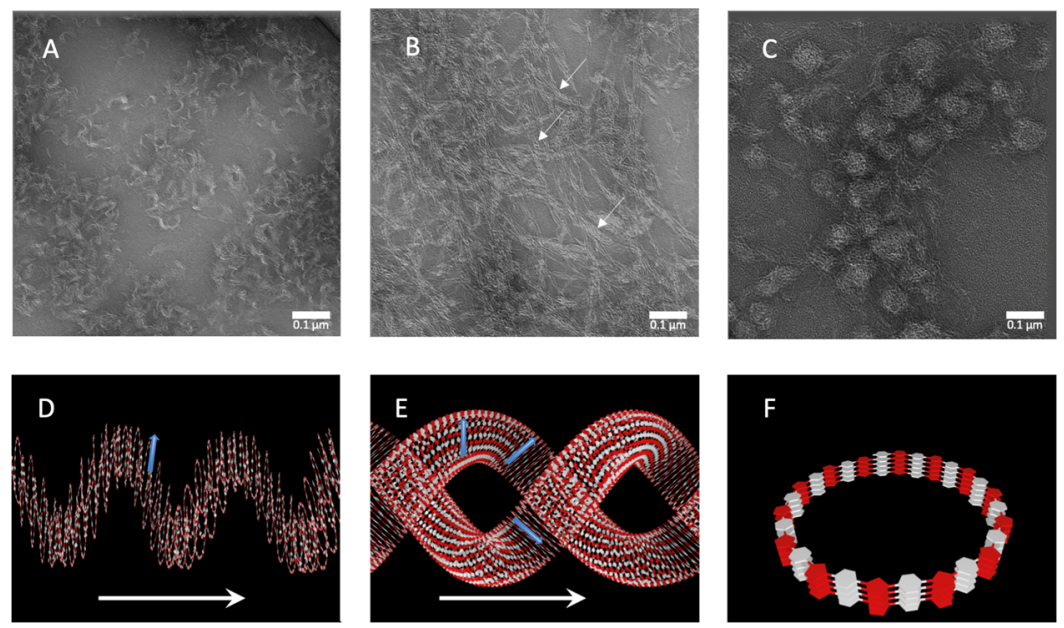

Figure 4. (A) TEM negative-stained image of $\mathrm{P}(p-\mathrm{AT}) \mathrm{S}-1$ at $15 \%$ water. (B) TEM negative-stained image of $\mathrm{P}(p$-AT) S-1 at $45 \%$ water. White arrows point at examples of the ubiquitous superhelices. (C) TEM negative-stained image of $\mathrm{P}(p-\mathrm{AT}) \mathrm{S}-\mathbf{1}$ at $90 \%$ water. (D) Cartoon representing the proposed structure of $\mathrm{P}(p-\mathrm{AT}) \mathrm{S}-\mathbf{1}$ at $15 \%$. The white arrow indicates shear direction. The blue arrow indicates chromophore transition moment polarizations relative to the long axis. (E) Cartoon representing the proposed structure of $\mathrm{P}(p$-AT) S-1 at $45 \%$. The white arrow indicates shear direction. Blue arrows indicate chromophore transition moment polarizations relative to the long axis. (F) Zoomed in details of one-folded $\mathrm{P}(p-\mathrm{AT}) \mathrm{S}-1$ chain at $45 \%$.

consistent with a semirigid "loose spring” chiral superstructure based on a fairly flat polymer pitch.

$\mathrm{P}(p-\mathrm{AT})$ Assemblies Formed at $20-50 \%$ Water. The LD signals for $\mathrm{P}(p$-AT $)$ with a water content above $20 \%$ are all a single positive band with the same shape, even though with differing magnitudes. The band maximum is red-shifted relative to the lower-water-content samples by about $8-10$ $\mathrm{nm}$ (Figure S8). The redshift is consistent with close $(\sim 3.4$ $\mathrm{nm}) \pi-\pi$ stacking interactions (as observed in e.g., DNA), as is the almost perfectly symmetrical exciton CD spectra (a positive Cotton effect for S-1) that develop.

The change in the sign of the LD signal is indicative of a different orientation of the aromatic chromophores. We tentatively assign this observation to the formation of elongated superstructures composed of tighter stacked helices that intertwine to form a "helix of helices" (cartoon in Figure $4 \mathrm{E}$ ), also known as superhelices, where the monomers are tilted to be less than $54.7^{\circ}$ from the long axis of the structure. This is in line with negative stain TEM images where twisting of the fibers can be observed (Figure 4B), although it is at the limit of the resolution of the images.

The $\mathrm{CD}$ and $\mathrm{LD}$ results can be combined to give more information about the local helical structure of the helices in this solvent regime. The $\mathrm{CD}$ signs and magnitudes for the degenerate coupling of helically oriented chromophores ${ }^{31}$ depend on the relative angles of EDTMs. The two energetically likely stacking options for the hexagon-pentagon $\pi$ systems are in either vertical stacks of identical units (Figure $4 \mathrm{~F}$ ) or stacks in alternating patterns (hexagon-pentagonhexagon, etc). The sign of the CD couplet together with the geometry required by the LD suggests that the $\mathbf{S}-\mathbf{1}$ polymer adopts either a right-handed helix with the aryl units (hexagons) vertically stacked on top of each other (Figure 4F) or a left-handed helix with alternating stacking of aryls (hexagons) and triazoles (pentagons). ${ }^{2} \mathrm{We}$ conclude that the former is more likely given the right-handed helix of the lowwater structures.

Approximating the superhelices as rigid structures of $\sim 1 \mu \mathrm{m}$ or longer (TEM of Figure 4B), the theoretical value for $S$ is $\sim 0.5-0.7 .^{49}$ The experimental $\mathrm{LD}^{\mathrm{r}}$ (from Figures 2 and 3 ) for $40 \%$ water is approximately 1 . This indicates that the average polymer monomer is tilted to about $40^{\circ}$ (or more precisely, the average $\cos 2 \alpha$ is 0.6$)$ from the helix axis. The cartoon of Figure $4 \mathrm{~F}$ shows how such a larger average tilt angle of the polymer planes can develop when a superhelix is formed (subsequent layers will tilt further). Thus, the structures we previously referred to as columns are superhelical chiral structures.

$\mathrm{P}(p-\mathrm{AT})$ Assemblies Formed at $>50 \%$ Water. The magnitude of LD signal intensity depends on the concentration of the analyte, its orientation parameter (which in turn depends on the length and rigidity of the structure), and the orientation of the transitions with respect to the helix axis. The gradual decrease in LD signal intensity at higher water percentages is caused by limited solubility of the tetra(ethylene glycol) appended backbone that leads to agglomeration into spherical structures, as seen in the TEM images, which do not give an LD signal. The CD indicates that the tightly twisted local packing of monomer units is retained in these structures.

$\mathrm{P}(p-\mathrm{AT})$ Assembly Stability in Shear Flow. As our understanding of the foldamers dictates that they form elongated structures composed of stacked helices, we were curious to determine their stability in shear flow. To that end, we tested the LD at different Couette shear flow rates from 0 to $5000 \mathrm{rpm}$. The principle of alignment via Couette flow is based on the fluid being confined between two cylinders, one of which rotates (in our case, the outer one) giving steady and unidirectional laminar flow. ${ }^{30,50,51}$ We tracked the LD signal at $310 \mathrm{~nm}$ and are clearly able to see the magnitude of the LD spectra increasing monotonically with rotation speed and hence shear magnitude (Figures S9 and S10). The lack of any degradation of signals at high shear indicates that the foldamer assembly is not destabilized by the higher shear rates we apply and that we have remained in the laminar flow regimes even for the lower-water-content samples. Thus, the unexpectedly rigid structures we observed (see above) for the loose helix regime $(10-20 \%$ water) are also stable pointing to larger 
complexity of the foldamer assemblies than originally concluded on the basis of $\mathrm{CD}$ and absorbance spectroscopy.

\section{CONCLUSIONS}

In this work, we have used the complementarity of absorbance, $\mathrm{CD}, \mathrm{LD}$, and TEM to investigate the structures formed from self-assembled foldamers designed to form helical structures. This, as far as we are aware, is the first time flow LD has been used to aid the characterization of synthetic polymer structures. We are able to estimate approximate orientations of the polymer EDTMs and hence of the polymer backbone and investigate the rigidity and stability of structures formed under different $\mathrm{DMF}$ /water ratios.

Based upon our absorbance, $\mathrm{CD}, \mathrm{LD}$, and EM studies, we therefore propose that from 0 to $9 \% \mathrm{H}_{2} \mathrm{O}$, the polymers are unfolded and unstructured. As the amount of water increases to $10-20 \%$, the structure develops into a loose helical structure that does not give rise to an excitonic CD signal but is chiral. The magnitude of the negative LD signal and the TEM images provide evidence that a stable fairly rigid supramolecular structure is already present at $10-20 \% \mathrm{H}_{2} \mathrm{O}$. The helices formed (right-handed for S-1) have a small pitch $\left(10-15^{\circ}\right.$ ) and assemble vertically and laterally, so the polymer monomers are approximately perpendicular to the fiber axis.

As the water concentration increases to above $20 \%$, the foldamers adopt a tighter wound helical conformation that gives rise to an excitonic Cotton effect in CD and evidence of twisted superhelical structures in TEM. The superhelix organization (see Figure 4E) tilts the planes of the monomer units, so the average EDTM is $54.7^{\circ}$ from the fiber axis with a resulting positive $\mathrm{LD}$ signal of larger intensity. Given the propensity of the $\mathbf{S - 1}$ monomer to form right-handed helices at a lower water content and the requirements of the sign of the Cotton effect and the sign of the LD, we deduce that the hexagons and pentagons locally stack vertically with their own kind (see Figure 4F).

The continued increase of water shows a drop in the LD signal, which from EM can be seen to be a result of spherical aggregate formation and loss of most of the high-aspect-ratio structures. The reason for this behavior is most likely the limited water solubility of the foldamers with a tetra(ethylene glycol) solubilizing moiety.

\section{ASSOCIATED CONTENT}

\section{SI Supporting Information}

The Supporting Information is available free of charge at https://pubs.acs.org/doi/10.1021/acsomega.1c06139.

Synthesis of backbone subunits; LD and CD studies; and TEM measurements (PDF)

\section{AUTHOR INFORMATION}

\section{Corresponding Authors}

Timothy R. Dafforn - School of Biosciences, University of Birmingham, Birmingham, West Midlands B15 2TT, U.K.; Email: t.r.dafforn@bham.ac.uk

Bert Klumperman - Department of Chemistry and Polymer Sciences, Stellenbosch University, Matieland 7602, South Africa; (i) orcid.org/0000-0003-1561-274X;

Email: bklump@sun.ac.za

\section{Authors}

Jake G. Carter - School of Chemistry, University of Birmingham, Birmingham, West Midlands B15 2TT, U.K.; School of Biosciences, University of Birmingham, Birmingham, West Midlands B15 2TT, U.K.

Rueben Pfukwa - Department of Chemistry and Polymer Sciences, Stellenbosch University, Matieland 7602, South Africa

Liam Riley - School of Biosciences, University of Birmingham, Birmingham, West Midlands B15 2TT, U.K.; School of Life Sciences, University of Warwick, Coventry CV4 7AL, U.K.

James H. R. Tucker - School of Chemistry, University of Birmingham, Birmingham, West Midlands B15 2TT, U.K.; (1) orcid.org/0000-0001-7645-0815

Alison Rodger - Department of Molecular Sciences, Macquarie University, Sydney, New South Wales 2109,

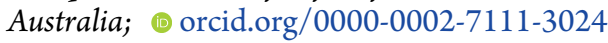

Complete contact information is available at:

https://pubs.acs.org/10.1021/acsomega.1c06139

\section{Author Contributions}

J.G.C. and R.P. contributed equally. The manuscript was written through contributions of all authors. All authors have given approval to the final version of the manuscript.

\section{Funding}

The authors wish to thank the Midlands Integrative Bioscience Training Partnership (MIBTP) for PhD studentship support to J.G.C. and L.R. (BB/R506175/1). We acknowledge the Midlands Regional Cryo-EM Facility, hosted at the Warwick Advanced Bioimaging Research Technology Platform, for use of the JEOL 2100Plus, supported by MRC award reference MC PC 17136. B.K. and R.P. acknowledge support by the South African Research Chairs Initiative of the Department of Science and Technology (DST) and National Research Foundation (NRF) of South Africa (grant no. 46855). This project has received funding from the European Union's Horizon 2020 research and innovation programme under the Marie Skłodowska-Curie grant agreement no. 778092.

Notes

The authors declare no competing financial interest.

\section{ACKNOWLEDGMENTS}

The Centre for Chemical and Materials Analysis at the University of Birmingham is acknowledged for technical support.

\section{REFERENCES}

(1) Le Bailly, B. A. F.; Clayden, J. Dynamic Foldamer Chemistry. Chem. Commun. 2016, 52, 4852-4863.

(2) Goodman, C. M.; Choi, S.; Shandler, S.; DeGrado, W. F. Foldamers as Versatile Frameworks for the Design and Evolution of Function. Nat. Chem. Biol. 2007, 3, 252-262.

(3) Yashima, E.; Ousaka, N.; Taura, D.; Shimomura, K.; Ikai, T.; Maeda, K. Supramolecular Helical Systems: Helical Assemblies of Small Molecules, Foldamers, and Polymers with Chiral Amplification and Their Functions. Chem. Rev. 2016, 116, 13752-13990.

(4) Guichard, G.; Huc, I. Synthetic Foldamers. Chem. Commun. 2011, 47, 5933.

(5) Saraogi, I.; Hamilton, A. D. Recent Advances in the Development of Aryl-Based Foldamers. Chem. Soc. Rev. 2009, 38, 1726.

(6) Zhang, D.-W.; Wang, H.; Li, Z.-T. Polymeric Tubular Aromatic Amide Helices. Macromol. Rapid Commun. 2017, 38, 1700179. 
(7) Liu, C.-Z.; Yan, M.; Wang, H.; Zhang, D.-W.; Li, Z.-T. Making Molecular and Macromolecular Helical Tubes: Covalent and Noncovalent Approaches. ACS Omega 2018, 3, 5165-5176.

(8) Xin, P.; Zhu, P.; Su, P.; Hou, J.-L.; Li, Z.-T. Hydrogen-Bonded Helical Hydrazide Oligomers and Polymer That Mimic the Ion Transport of Gramicidin A. J. Am. Chem. Soc. 2014, 136, 1307813081.

(9) Ferrand, Y.; Kendhale, A. M.; Kauffmann, B.; Grélard, A.; Marie, C.; Blot, V.; Pipelier, M.; Dubreuil, D.; Huc, I. Diastereoselective Encapsulation of Tartaric Acid by a Helical Aromatic Oligoamide. J. Am. Chem. Soc. 2010, 132, 7858-7859.

(10) Juwarker, H.; Suk, J.-m.; Jeong, K.-S. Foldamers with Helical Cavities for Binding Complementary Guests. Chem. Soc. Rev. 2009, $38,3316$.

(11) Lang, C.; Li, W.; Dong, Z.; Zhang, X.; Yang, F.; Yang, B.; Deng, X.; Zhang, C.; Xu, J.; Liu, J. Biomimetic Transmembrane Channels with High Stability and Transporting Efficiency from Helically Folded Macromolecules. Angew. Chem., Int. Ed. 2016, 55, 9723-9727.

(12) Pfukwa, R.; Kouwer, P. H. J.; Rowan, A. E.; Klumperman, B. Templated Hierarchical Self-Assembly of Poly(p-Aryltriazole) Foldamers. Angew. Chem., Int. Ed. 2013, 52, 11040-11044.

(13) Ousaka, N.; Yamaguchi, T.; Yashima, E. Remarkable Enhancement of Stability and Helix-Sense Excess of Oligo(Phenylene Ethynylene) Foldamers Assisted by Linking with Achiral (Metallo)Salen Tethers and Their Application to Asymmetric Catalysis. Chem. Lett. 2014, 43, 512-514.

(14) Zheng, L.; Zheng, D.; Wang, Y.; Yu, C.; Zhang, K.; Jiang, H. Chiral Bisphosphine Ligands Based on Quinoline Oligoamide Foldamers: Application in Asymmetric Hydrogenation. Org. Biomol. Chem. 2019, 17, 9573-9577.

(15) Nelson, J. C.; Saven, J. G.; Moore, J. S.; Wolynes, P. G. Solvophobically Driven Folding of Nonbiological Oligomers. Science 1997, 277, 1793-1796.

(16) Hamuro, Y.; Geib, S. J.; Hamilton, A. D. Oligoanthranilamides. Non-Peptide Subunits That Show Formation of Specific Secondary Structure. J. Am. Chem. Soc. 1996, 118, 7529-7541.

(17) Berl, V.; Huc, I.; Khoury, R. G.; Lehn, J.-M. Helical Molecular Programming: Folding of Oligopyridine-Dicarboxamides into Molecular Single Helices. Chem.-Eur. J. 2001, 7, 2798-2809.

(18) Petitjean, A.; Cuccia, L. A.; Lehn, J.-M.; Nierengarten, H.; Schmutz, M. Cation-Promoted Hierarchical Formation of Supramolecular Assemblies of Self-Organized Helical Molecular Components. Angew. Chem., Int. Ed. 2002, 41, 1195-1198.

(19) Brioche, J.; Pike, S. J.; Tshepelevitsh, S.; Leito, I.; Morris, G. A.; Webb, S. J.; Clayden, J. Conformational Switching of a Foldamer in a Multicomponent System by PH-Filtered Selection between Competing Noncovalent Interactions. J. Am. Chem. Soc. 2015, 137, 66806691

(20) Prince, R. B.; Saven, J. G.; Wolynes, P. G.; Moore, J. S. Cooperative Conformational Transitions in Phenylene Ethynylene Oligomers: Chain-Length Dependence. J. Am. Chem. Soc. 1999, 121, 3114-3121.

(21) Zhang, P.; Zhang, L.; Wang, H.; Zhang, D.-W.; Li, Z.-T. Helical Folding of an Arylamide Polymer in Water and Organic Solvents of Varying Polarity. Polym. Chem. 2015, 6, 2955-2961.

(22) Gin, M. S.; Yokozawa, T.; Prince, R. B.; Moore, J. S. Helical Bias in Solvophobically Folded Oligo(Phenylene Ethynylene)s. J. Am. Chem. Soc. 1999, 121, 2643-2644.

(23) Brunsveld, L.; Zhang, H.; Glasbeek, M.; Vekemans, J. A. J. M.; Meijer, E. W. Hierarchical Growth of Chiral Self-Assembled Structures in Protic Media †. J. Am. Chem. Soc. 2000, 122, 6175-6182. (24) Jeon, H.-G.; Jung, J. Y.; Kang, P.; Choi, M.-G.; Jeong, K.-S. Folding-Generated Molecular Tubes Containing One-Dimensional Water Chains. J. Am. Chem. Soc. 2016, 138, 92-95.

(25) Prince, R. B.; Brunsveld, L.; Meijer, E. W.; Moore, J. S. Twist Sense Bias Induced by Chiral Side Chains in Helically Folded Oligomers. Angew. Chem., Int. Ed. 2000, 39, 228-230.

(26) Prince, R. B.; Moore, J. S.; Brunsveld, L.; Meijer, E. W. Cooperativity in the Folding of Helical M-Phenylene Ethynylene
Oligomers Based upon the Sergeants-and-Soldiers Principle. Chem.Eur. J. 2001, 7, 4150-4154.

(27) Cuccia, L. A.; Lehn, J.-M.; Homo, J.-C.; Schmutz, M. Encoded Helical Self-Organization and Self-Assembly into Helical Fibers of an Oligoheterocyclic Pyridine - Pyridazine Molecular Strand. Angew. Chem., Int. Ed. 2000, 39, 233-237.

(28) Creager, A. N. H.; Scholthof, K.-B. G.; Citovsky, V.; Scholthof, H. B. Tobacco Mosaic Virus: Pioneering Research for a Century. Plant Cell 1999, 11, 301-308.

(29) Hicks, M. R.; Kowałski, J.; Rodger, A. LD Spectroscopy of Natural and Synthetic Biomaterials. Chem. Soc. Rev. 2010, 39, 3380.

(30) Rodger, A.; Marrington, R.; Geeves, M. A.; Hicks, M.; de Alwis, L.; Halsall, D. J.; Dafforn, T. R. Looking at Long Molecules in Solution: What Happens When They Are Subjected to Couette Flow? Phys. Chem. Chem. Phys. 2006, 8, 3161.

(31) Nordén, B.; Rodger, A.; Dafforn, T. Linear Dichroism and Circular Dichroism: A Textbook on Polarized-Light Spectroscopy; Royal Society of Chemistry: Cambridge, 2010.

(32) Pacheco-Gómez, R.; Kraemer, J.; Stokoe, S.; England, H. J.; Penn, C. W.; Stanley, E.; Rodger, A.; Ward, J.; Hicks, M. R.; Dafforn, T. R. Detection of Pathogenic Bacteria Using a Homogeneous Immunoassay Based on Shear Alignment of Virus Particles and Linear Dichroism. Anal. Chem. 2012, 84, 91.

(33) Carr-Smith, J.; Pacheco-Gómez, R.; Little, H. A.; Hicks, M. R.; Sandhu, S.; Steinke, N.; Smith, D. J.; Rodger, A.; Goodchild, S. A.; Lukaszewski, R. A.; Tucker, J. H. R.; Dafforn, T. R. Polymerase Chain Reaction on a Viral Nanoparticle. ACS Synth. Biol. 2015, 4, 13161325.

(34) Marrington, R.; Dafforn, T. R.; Halsall, D. J.; MacDonald, J. I.; Hicks, M.; Rodger, A. Validation of New Microvolume Couette Flow Linear Dichroism Cells. Analyst 2005, 130, 1608.

(35) Clack, B. A.; Gray, D. M. Flow Linear Dichroism Spectra of Four Filamentous Bacteriophages: DNA and Coat Protein Contributions. Biopolymers 1992, 32, 795-810.

(36) Rittman, M.; Hoffmann, S. V.; Gilroy, E.; Hicks, M. R.; Finkenstadt, B.; Rodger, A. Probing the Structure of Long DNA Molecules in Solution Using Synchrotron Radiation Linear Dichroism. Phys. Chem. Chem. Phys. 2012, 14, 353-366.

(37) Rodger, A.; Dorrington, G.; Ang, D. L. Linear Dichroism as a Probe of Molecular Structure and Interactions. Analyst 2016, 141, 6490-6498.

(38) Razmkhah, K.; Chmel, N. P.; Gibson, M. I.; Rodger, A. Oxidized Polyethylene Films for Orienting Polar Molecules for Linear Dichroism Spectroscopy. Analyst 2014, 139, 1372-1382.

(39) Tridgett, M.; Moore-Kelly, C.; Duprey, J.-L. H. A.; Iturbe, L. O.; Tsang, C. W.; Little, H. A.; Sandhu, S. K.; Hicks, M. R.; Dafforn, T. R.; Rodger, A. Linear Dichroism of Visible-Region Chromophores Using M13 Bacteriophage as an Alignment Scaffold. RSC Adv. 2018, 8, 29535-29543.

(40) Nordén, B.; Kurucsev, T. Analysing DNA Complexes by Circular and Linear Dichroism. J. Mol. Recognit. 1994, 7, 141-155.

(41) Chou, P.-J.; Johnson, W. C. Base Inclinations in Natural and Synthetic DNAs. J. Am. Chem. Soc. 1993, 115, 1205-1214.

(42) Norden, B.; Kubista, M.; Kurucsev, T. Linear Dichroism Spectroscopy of Nucleic Acids. Q. Rev. Biophys. 1992, 25, 51.

(43) Hicks, M. R.; Rodger, A.; Lin, Y.-p.; Jones, N. C.; Hoffmann, S. V.; Dafforn, T. R. Rapid Injection Linear Dichroism for Studying the Kinetics of Biological Processes. Anal. Chem. 2012, 84, 6561-6566.

(44) Bromley, E. H. C.; Channon, K. J.; King, P. J. S.; Mahmoud, Z. N.; Banwell, E. F.; Butler, M. F.; Crump, M. P.; Dafforn, T. R.; Hicks, M. R.; Hirst, J. D.; Rodger, A.; Woolfson, D. N. Assembly Pathway of a Designed $\alpha$-Helical Protein Fiber. Biophys. J. 2010, 98, 1668-1676.

(45) Marshall, K. E.; Hicks, M. R.; Williams, T. L.; Hoffmann, S. V.; Rodger, A.; Dafforn, T. R.; Serpell, L. C. Characterizing the Assembly of the Sup35 Yeast Prion Fragment, GNNQQNY: Structural Changes Accompany a Fiber-to-Crystal Switch. Biophys. J. 2010, 98, 330-338.

(46) Ali, A.; Little, H. A.; Carter, J. G.; Douglas, C.; Hicks, M. R.; Kenyon, D. M.; Lacomme, C.; Logan, R. T.; Dafforn, T. R.; Tucker, J. H. R. Combining Bacteriophage Engineering and Linear Dichroism 
Spectroscopy to Produce a DNA Hybridisation Assay. RSC Chem. Biol. 2020, 1, 449.

(47) Rodger, P. M.; Montgomery, C.; Costantini, G.; Rodger, A. Morphology, Energetics and Growth Kinetics of Diphenylalanine Fibres. Phys. Chem. Chem. Phys. 2021, 23, 4597-4604.

(48) Katan, C.; Savel, P.; Wong, B. M.; Roisnel, T.; Dorcet, V.; Fillaut, J.-L.; Jacquemin, D. Absorption and Fluorescence Signatures of 1,2,3-Triazole Based Regioisomers: Challenging Compounds for TD-DFT. Phys. Chem. Chem. Phys. 2014, 16, 9064-9073.

(49) McLachlan, J. R. A.; Smith, D. J.; Chmel, N. P.; Rodger, A. Calculations of Flow-Induced Orientation Distributions for Analysis of Linear Dichroism Spectroscopy. Soft Matter 2013, 9, 4977.

(50) Taylor, G. I., VIII Stability of a Viscous Liquid Contained between Two Rotating Cylinders. Philos. Trans. R. Soc., A 1923, 223, 289-343.

(51) Coles, D. Transition in Circular Couette Flow. J. Fluid Mech. $1965,21,385-425$. 